\title{
Targeting of IgMк Antibodies to Oligodendrocytes Promotes CNS Remyelination
}

\author{
Kunihiko Asakura, ${ }^{1,2}$ David J. Miller, ${ }^{2}$ Larry R. Pease, ${ }^{2}$ and Moses Rodriguez ${ }^{1,2}$ \\ Departments of ${ }^{1}$ Neurology and ${ }^{2} /$ mmunology, Mayo Clinic and Foundation, Rochester, Minnesota 55905
}

We previously identified the remyelinating activity of a natural IgMк oligodendrocyte-reactive autoantibody (SCH94.03), using a virus-induced murine model of multiple sclerosis. We now describe a second mouse $\lg \mathrm{M} \kappa$ monoclonal antibody (mAb) (SCH79.08) raised against normal mouse spinal cord homogenate, which reacts with myelin basic protein and also promotes remyelination. Because these two mAbs recognize different oligodendrocyte antigens, several previously identified oligodendrocyte-reactive IgM $\kappa$ mAbs (O1, O4, A2B5, and HNK1 ), each with distinct antigen specificities, were evaluated and found to promote remyelination. In contrast, IgM $\mathrm{mAbs}$ that did not bind to oligodendrocytes showed no remyelination. One of these, $\mathrm{CH}_{12} \lg \mathrm{M}_{\kappa} \mathrm{mAb}$, which shares variable region cDNA sequences with $\mathrm{SCH} 94.03$ except for amino acid differences in the complementarity-determining region 3 in both heavy and light chains, did not bind to oligodendrocytes and did not promote remyelination. The fact that multiple oligodendrocytereactive antibodies with distinct antigen reactivities induce remyelination argues against direct activation by a unique cell surface receptor. These findings are most consistent with the hypothesis that the binding of mAbs to oligodendrocytes in the lesions induces myelin repair via indirect immune effector mechanisms initiated by the $\mu$-chain. Importantly, these studies indicate that oligodendrocyte-reactive natural autoantibodies may provide a powerful and novel therapeutic means to induce remyelination in multiple sclerosis patients.

Key words: Theiler's virus; oligodendrocytes; demyelination; natural autoantibody; remyelination; multiple sclerosis; immunoglobulin
Demyelination in association with inflammation is the primary structural abnormality in multiple sclerosis (MS). Spontaneous remyelination is limited in the CNS, in part because oligodendrocytes are considered to be postmitotic cells. However, spontaneous remyelination is observed at the edge of MS plaques (Prineas and Connell, 1979). Studies have shown that oligodendrocyte/type-2 astrocyte (O-2A) progenitor cells persist in the adult CNS and proliferate (Wolswijk and Noble, 1989; Armstrong et al., 1992). Alternatively, it has been shown that mature oligodendrocytes can be induced to generate new myelin under the influence of neurons (Wood and Bunge, 1991). Basic fibroblast growth factor induces mature oligodendrocytes to reenter the cell cycle (Fressinaud et al., 1993; Grinspan et al., 1993) and converts the cells to a novel phenotype (Bansal and Pfeiffer, 1997), providing another cellular mechanism for remyelination.

One of the major goals for the treatment of MS is to develop strategies to promote remyelination. One strategy shown in vivo to enhance endogenous myelination has been the use of natural germline antibodies that react to CNS antigens (Miller et al., 1994). This approach is particularly attractive because it rapidly can be translated from the bench to the bedside as a therapy for human MS (Noseworthy et al., 1994; Fazekas et al., 1997).

Received June 4, 1998; revised July 21, 1998; accepted July 21, 1998.

These studies were supported by National Institutes of Health Grant R01 NS24180. We appreciate the generous contributions of Mr. and Mrs. Eugene Applebaum to this project. We thank Dorian McGavern for a critical review of this manuscript and Kevin Pavelko and Laurie Zoecklein for technical assistance.

Correspondence should be addressed to Dr. Moses Rodriguez, Department of Immunology, Mayo Clinic, 200 First Street SW, Rochester, MN 55905.

Dr. Miller's present address: University of Wisconsin Hospitals and Clinics, Madison, WI 53792.

Copyright (C) 1998 Society for Neuroscience $\quad 0270-6474 / 98 / 187700-09 \$ 05.00 / 0$
We demonstrated that a mouse monoclonal antibody (mAb) raised against normal mouse spinal cord homogenate $(\mathrm{SCH})$, designated SCH94.03, enhanced CNS remyelination in the Theiler's murine encephalomyelitis virus (TMEV) model of MS (Miller et al., 1994). SCH94.03 belongs to the IgM $\kappa$ subclass, is highly polyreactive against known and unknown protein antigens including cytoskeletal proteins, and is encoded by unmutated Ig germline genes, confirming that SCH94.03 is a natural autoantibody (Miller and Rodriguez, 1995a; Asakura et al., 1996a). Of unique importance, SCH94.03 recognizes an unidentified surface antigen on oligodendrocytes (Asakura et al., 1996b), providing a potential target for the mechanism of action of this antibody.

Two major hypotheses have been proposed by which SCH94.03 promotes remyelination. (1) The mAb may bind to a unique receptor on the surface of oligodendrocytes to induce myelination. This hypothesis would predict that only a limited repertoire of Abs with unique specificity would function for myelin repair. (2) The mAb may work by binding to damaged oligodendrocytes and/or myelin, which triggers a cascade of events by other resident CNS cells (i.e., astrocytes, microglia, or neurons) and in turn enhances myelin repair. An attractive hypothesis is that binding to damaged oligodendrocytes and myelin may enhance the opsonization and clearing of CNS debris by macrophages, thus allowing for the normal process of remyelination to ensue. This hypothesis would predict that many polyreactive autoantibodies with specificity to oligodendrocytes and/or myelin would be effective. To address these hypotheses and the mechanism for Ab-mediated CNS remyelination, we set out to identify additional mAbs that promote CNS remyelination in TMEV model and compared their specificities with SCH94.03. In addition, we tested the remyelination-promoting activity of well recognized oligodendrocyte-reactive mAbs O1, O4, A2B5, and HNK-1, 
which previously were shown to have genotypic or phenotypic features of natural autoantibodies (Asakura et al., 1995).

\section{MATERIALS AND METHODS}

$m A b$ production and screening. Hybridomas were generated and screened as described (Miller et al., 1994). SJL/J mice were immunized with normal mouse SCH in incomplete Freund's adjuvant, and their splenocytes were fused with NS-1 myeloma cell. Hybridoma supernatants that showed high binding to SCH by ELISA were screened further for their ability to promote remyelination in the TMEV model. Therefore, the Abs were screened on the basis of their ability to promote CNS remyelination rather than for a unique antigen specificity. IgM $\kappa$ Abs were purified from hybridoma culture supernatants by ammonium sulfate precipitation and dialysis against PBS plus low-ionic-strength precipitation or by affinity chromatography, using goat anti-mouse $\operatorname{IgM~Ab}(\mu-$ chain-specific; Jackson ImmunoResearch, West Grove, PA) bound to carbonyldiimidazole-activated cross-linked agarose (Reacti-Gel $6 \times$ matrix, Pierce, Rockford, IL).

Hybridomas and $m A b$ preparation. Hybridomas $\mathrm{A} 2 \mathrm{~B} 5, \mathrm{HNK}-1$, and R24 were purchased from American Type Culture Collection (ATCC, Rockville, MD). Hybridomas O1 and O4 were a gift from Dr. S. E. Pfeiffer (University of Connecticut, Farmington, CT). These hybridomas were cultured in RPMI 1640 supplemented with $10 \%$ fetal calf serum (HyClone, Logan, UT) and $2 \times 10^{-2} \mathrm{~mm} \beta$-mercaptoethanol. B-cell lymphoma CH12 (CH12.Lx) was provided by Dr. G. Haughton (University of North Carolina, Chapel Hill, NC). To obtain secreted IgM from CH12 lymphoma, we stimulated CH12.Lx cells with $50 \mu \mathrm{g} / \mathrm{ml}$ of lipopolysaccharide (Sigma, St. Louis, MO). mAbs O1, O4, and HNK-1 were purified from hybridoma culture supernatant by ammonium sulfate precipitation and dialysis against PBS plus low-ionic-strength precipitation. mAbs A2B5 and CH12 were purified by affinity chromatography. R24 was purified by protein A column. The purity of these mAbs was examined by SDS-polyacrylamide gel electrophoresis, and the immunoreactivity of these $\mathrm{mAbs}$ was examined by the immunostaining of rat oligodendrocytes. Control hybridoma XMMEN-OE5 producing antibacterial lipopolysaccharide $\operatorname{IgM} \kappa \mathrm{Ab}$ was purchased from ATCC. Clarified ascites of control ABPC22 IgM $\kappa$ mAb were purchased from Sigma. Both $\operatorname{IgM} \kappa \mathrm{mAbs}$ were purified by affinity chromatography, using goat anti-mouse IgM Ab. The purity of the mAbs was confirmed by SDSpolyacrylamide gel electrophoresis.

Virus and animals. The Daniel's strain of TMEV was used for these experiments. Female SJL/J mice from the Jackson Laboratories (Bar Harbor, ME) were used after 1 week of rest. Mice from 4 to 6 weeks of age were injected intracerebrally with $2 \times 10^{5}$ plaque-forming units ( $\mathrm{pfu}$ ) of TMEV in a $10 \mu$ l volume. The handling of all animals was in accordance with the institutional guidelines prescribed by National Institutes of Health and Mayo Clinic.

$m A b$ treatment and quantitative morphometry of remyelination. Chronically infected mice (5-6 months after infection) were given intraperitoneal injections of mAb twice weekly for 5 weeks ( $50 \mu \mathrm{g} /$ injection). The total dose of each $\mathrm{Ab}$ was $0.5 \mathrm{mg}$. The mice were killed 2 weeks after the completion of mAb treatment. Light microscopic sections were prepared as described (Miller et al., 1994). Mice were anesthetized with pentobarbital, exsanguinated by cardiac puncture, and fixed by intracardiac perfusion with Trump's fixative (phosphate-buffered $4 \%$ formaldehyde containing $1.5 \%$ glutaraldehyde, $\mathrm{pH}$ 7.2). The entire spinal cord was removed and sectioned into $1 \mathrm{~mm}$ transverse blocks. Every third block was post-fixed in 1\% osmium tetroxide and embedded in Araldite (Polysciences, Warrington, PA). One micrometer sections were cut and stained with $p$-phenylenediamine. Ten spinal cord sections of each mouse were examined. The total areas of white matter, demyelination, and remyelination on each section were quantitated by a Zeiss (Oberkochen, Germany) interactive digital analysis system. The area of demyelination was characterized by cellular infiltrates, macrophages engulfing myelin debris, and naked axons. Abnormally thin myelin sheaths relative to axon diameter were used as the criterion for remyelination by oligodendrocytes. Those remyelinated fibers were identified readily (as shown in Fig. 1 ). Remyelinated areas were defined as a cluster of at least 10 remyelinated fibers. Spontaneous remyelination by Schwann cells also was present rarely in the spinal cord lesions (Miller and Rodriguez, 1995b). Remyelination by Schwann cell was characterized by abnormally thick myelin sheaths relative to axon diameter, with a one-to-one relationship between axons and Schwann cells. These peripheral-type remyelinated areas were excluded from the quantitation. The quantitation was done on coded sections without previous knowledge of the treatment to avoid bias. Statistical comparison between groups in the extent of demyelination and remyelination was performed with an unpaired Student's $t$ test.

Cell culture and immunocytochemistry. Oligodendrocytes, astrocytes, and microglia were isolated from telencephalon of newborn Sprague Dawley rats as described (Asakura et al., 1996b). Abs were diluted in PBS. Surface staining was performed at $4^{\circ} \mathrm{C}$ for 15 min on unfixed cells after they were blocked with PBS containing $3 \%$ normal goat serum (NGS). Cytoplasmic antigen staining was performed on cells fixed for 10 min at $4^{\circ} \mathrm{C}$ with $2 \%$ paraformaldehyde and treated for $5 \mathrm{~min}$ at room temperature with $0.1 \%$ Triton X-100 in PBS, followed by blocking with $3 \%$ NGS in PBS. After incubation with the primary Abs and the secondary FITC-conjugated anti-mouse IgM ( $\mu$-chain-specific) Ab (Jackson ImmunoResearch), the slides were mounted in MOWIOL (Aldrich Chemical, Milwaukee, WI) containing 2.5\% 1,4-diazobicyclo-[2.2.2]octane (DABCO; Sigma) and were viewed with an epifluorescent microscope.

Immunohistochemistry. Fresh-frozen sections $(10 \mu \mathrm{m})$ were prepared from various organs of neonatal (postnatal days 7 and 14) rats. Freshfrozen sections were immunostained with primary Abs and then were fixed with $4 \%$ paraformaldehyde or were lightly fixed with acetone and then were immunostained with primary Abs. Bound Abs were detected by fluorochrome-conjugated secondary $\mathrm{Ab}$ or by the avidin-biotin immunoperoxidase technique, using the Vectastain ABC kit (Vector Laboratories, Burlingame, CA). MOPC104E (Sigma) mouse IgM mAb was used as an isotype control.

Direct ELISA. Protein antigens, including human red blood cell (RBC) spectrin, bovine myosin (heavy chain), mouse albumin, mouse hemoglobin, mouse transferrin, hen egg lysozyme (HEL), rabbit actin, rabbit myelin basic protein (MBP), and keyhole limpet hemocyanin (KLH), were purchased from Sigma. Proteins were tested for purity by SDSpolyacrylamide gel electrophoresis. All chemical haptens were purchased from Sigma and coupled to bovine serum albumin (BSA) (Miller and Rodriguez, 1995a). Protein antigens were used at $5 \mu \mathrm{g} / \mathrm{ml}$ and haptens at $2 \mu \mathrm{M}$. The proteins and hapten-BSA antigens were coated onto polystyrene or polyvinyl chloride microtiter plates in $0.1 \mathrm{M}$ carbonate buffer, $\mathrm{pH}$ 9.5, for $18 \mathrm{hr}$ at $4^{\circ} \mathrm{C}$. Coated plates were blocked with PBS containing $5 \%$ nonfat dry milk and $0.05 \%$ Tween 20 for $2 \mathrm{hr}$ at room temperature and were incubated with mAbs diluted in blocking buffer for $4 \mathrm{hr}$ at room temperature. TEPC183 (Sigma) and XMMEN-OE5 IgM $\kappa$ mAbs were used as isotype control Abs. Bound IgM was detected with biotinylated goat anti-mouse IgM ( $\mu$-chain-specific; Jackson ImmunoResearch), followed by alkaline phosphatase conjugated to streptavidin, with $p$-nitrophenylphosphate as the chromogenic substrate. Absorbance was determined at $405 \mathrm{~nm}$.

Immunoblotting. Purified TMEV (Njenga et al., 1996) and rabbit MBP (Sigma) were separated by SDS-polyacrylamide gel electrophoresis on $15 \%$ acrylamide gels. Proteins were transferred to a nitrocellulose membrane by electroblotting. The membrane was blocked with Tris-buffered saline containing 5\% nonfat dry milk and $0.03 \%$ Tween 20 for $2 \mathrm{hr}$ at room temperature. The membrane was incubated with $\mathrm{SCH} 79.08$, SCH94.03, O1， O4, A2B5, HNK-1, CH12， R24, control IgM (MOPC104E), rabbit polyclonal anti-TMEV (1:2000; Njenga et al., 1996), and rabbit polyclonal anti-MBP (1:200; Dako, Carpinteria, CA) Abs for $4 \mathrm{hr}$ at room temperature. SCH79.08, SCH94.03, O1, O4, A2B5, HNK-1, CH12, R24, and control IgM were used at the same concentration $(10 \mu \mathrm{g} / \mathrm{ml})$. Bound Abs were detected with biotinylated secondary Abs (Jackson ImmunoResearch) and alkaline phosphatase-conjugated streptavidin, using 5-bromo-4-chloro-3-indolyl phosphate and nitroblue tetrazolium (BCIP/NBT).

\section{RESULTS}

\section{IgMк mAb SCH79.08 promotes CNS remyelination}

After cell fusion and cloning, a large panel of mAbs $(\sim 100)$ was screened by ELISA. One mAb, designated SCH79.08 and belonging to the $\operatorname{IgM} \kappa$ subclass, showed significant binding to $\mathrm{SCH}$ by ELISA. SJL/J mice chronically infected with TMEV and treated with SCH79.08 showed significantly greater CNS remyelination than animals treated with PBS or isotype-matched control mAb (Table 1). On average, $\sim 20 \%$ of the total demyelinated area showed CNS remyelination in mice treated with SCH79.08 $(p<$ $0.05)$, as compared with $2-5 \%$ in animals treated with PBS or isotype-matched mAb, respectively. Treatment with SCH79.08 
Table 1. Enhancement of CNS remyelination by SCH79.08

\begin{tabular}{|c|c|c|c|c|c|}
\hline Treatment & $\begin{array}{l}\text { Number } \\
\text { of mice }\end{array}$ & $\begin{array}{l}\text { Area of white } \\
\text { matter }\left(\mathrm{mm}^{2}\right)\end{array}$ & $\begin{array}{l}\text { Area of demyelinated } \\
\text { lesion }\left(\mathrm{mm}^{2}\right)\end{array}$ & $\begin{array}{l}\text { Area of CNS-type } \\
\text { remyelination }\left(\mathrm{mm}^{2}\right)\end{array}$ & $\begin{array}{l}\text { Area of CNS-type } \\
\text { remyelination/area } \\
\text { of lesions }(\%)\end{array}$ \\
\hline SCH79.08 & 15 & $8.42 \pm 0.33$ & $1.01 \pm 0.16$ & $0.20 \pm 0.05$ & $20.2 \pm 4.7$ \\
\hline ABPC22 & 7 & $8.06 \pm 0.46$ & $1.05 \pm 0.22$ & $0.05 \pm 0.01$ & $4.9 \pm 1.4$ \\
\hline PBS & 6 & $8.89 \pm 0.26$ & $1.01 \pm 0.21$ & $0.03 \pm 0.01$ & $2.4 \pm 0.8$ \\
\hline
\end{tabular}

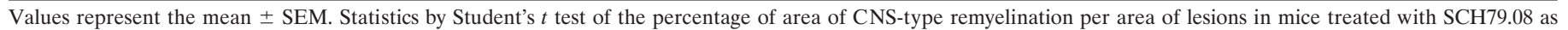
compared with mice treated with ABPC22 (isotype control) or PBS revealed $p<0.05$.

had no effect on the extent of demyelination (Table 1). Remyelinated lesions were characterized by hundreds of axons with thin myelin sheaths and a relative absence of inflammatory cells or macrophages (Fig. 1). In contrast, most lesions in mice treated with the control $\operatorname{IgM} \kappa \mathrm{mAb}$ ABPC22 or PBS had few, if any, remyelinated axons, and the lesions contained intense inflammation and macrophage infiltration (Fig. 1).

\section{mAb SCH79.08 is polyreactive and reacts with MBP}

To characterize the antigens recognized by $\mathrm{SCH} 79.08$, we performed immunochemistry, ELISA, and Western blotting. By immunocytochemical study, SCH79.08 strongly stained the cytoplasmic structure of most of the cultured cells (Fig. 2D). The pattern of reactivity resembled the staining with SCH94.03 (Fig. $2 B$ ), presumably to a cytoskeletal protein. In contrast to the live surface staining of oligodendrocytes, oligodendrocytes at specific stages of differentiation did not react on the surface with SCH79.08 (Fig. 2C). Immunohistochemical staining of freshfrozen rat tissue sections showed that $\mathrm{SCH} 79.08$ is reactive to multiple organs, including brain (predominantly white matter glial cell population), small intestine (lamina propria), and kidney (mesangial cells). These results are consistent with the conclusion that $\mathrm{SCH} 79.08$ is polyreactive.

To assess further the polyreactivity of SCH79.08, we performed ELISA, using a panel of protein antigens and chemical haptens. SCH79.08 showed prominent reactivity toward RBC spectrin, but it also reacted with rabbit actin, rabbit MBP, KLH, and mouse hemoglobin (Fig. $3 A$ ). SCH79.08 also showed reactivity toward multiple chemical haptens, including phenyloxazolone (PhoX), (4-hydroxy-3-nitrophenyl)acetyl (NP), and azophenyltrimethyl ammonium (TMA) (Fig. 3B). No reactivity was detected with the carrier protein BSA. Control $\operatorname{IgM} \mathrm{mAb}$ did not react with any of the protein antigens and chemical haptens that were tested (Fig. 3C,D). By Western blotting, SCH79.08 showed reactivity against the 21.5 and $18.5 \mathrm{kDa}$ isoforms of rabbit $\mathrm{MBP}$, confirming the ELISA result (Fig. 4B).

To exclude the possibility that remyelination was the consequence of SCH79.08 reacting with TMEV, we performed Western blotting, using purified TMEV. SCH79.08 did not react with any of the known TMEV capsid proteins (Fig. 4A).

\section{Variable region cDNA sequences of $\mathrm{SCH} 79.08$ are different from those of the prototypic remyelination- promoting Ab SCH94.03}

Despite binding similarities, variable region cDNA sequences of SCH79.08 were completely different from those of SCH94.03 (Miller and Rodriguez, 1995a) (cDNA sequences of SCH79.08 are available from the GenBank database under accession numbers U91317 and U92070). SCH79.08 VH belonged to the VH558 family. The D segment was derived from the germline DQ52 gene. The $\mathrm{JH}$ region was identical to the $\mathrm{JH} 2$ germline gene. The
$\mathrm{V} \kappa$ segment for the light chain belonged to the $\mathrm{V} \kappa 24$ family, whereas the $\mathrm{J} \kappa$ segment was identical to the $\mathrm{J} \kappa 5$ germline gene.

\section{Multiple oligodendrocyte-reactive IgMк mAbs with unique antigen specificities promote CNS remyelination}

Of interest, SCH94.03 and SCH79.08 have important similarities with a number of well characterized oligodendrocyte-reactive mAbs. Mouse IgM $\kappa$ mAbs O1, O4 (Sommer and Schachner, 1981), A2B5 (Eisenbarth et al., 1979), and HNK-1 (Abo and Balch, 1981) recognize unique differentiation stage-specific surface antigens on oligodendrocytes. O1 recognizes multiple lipid antigens, including galactocerebroside, monogalactosyl-diglyceride, and psychosine (Bansal et al., 1989); O4 recognizes proligodendroblast antigen and sulfatide (Bansal et al., 1989, 1992); and A2B5 recognizes ganglioside GQ1c and other gangliosides (Kasai and Yu, 1983; Fredman et al., 1984). The carbohydrate epitope on myelin-associated glycoprotein (MAG) appears to be the principal antigen recognized by HNK-1 (McGarry et al., 1983). This carbohydrate epitope recognized by HNK-1 is also present in other cell adhesion molecules in the nervous system. Similar to $\mathrm{SCH} 94.03$ and $\mathrm{SCH} 79.08$, these mAbs all belong to the $\operatorname{IgM} \kappa$ subclass, are polyreactive, recognize distinct antigens on oligodendrocytes, and recognize intracellular structures of many cell types. In addition, variable region cDNA sequences of these $\mathrm{mAbs}$ have indicated minimal mutations from the germline Ig genes, a characteristic feature of natural autoantibodies (Asakura et al., 1995).

On the basis of these striking similarities, we tested the therapeutic efficacy of oligodendrocyte-reactive mAbs O1, O4, A2B5, and HNK-1 in the TMEV model. A mouse IgG3 mAb R24, which recognizes ganglioside GD3 expressed on O-2A progenitor cells, and an irrelevant mouse IgM $\kappa$ mAb (XMMEN-OE5) without reactivity to oligodendrocytes also were tested to determine whether Ig isotype and specificity to a unique oligodendrocyte differentiation stage were critical for function. SJL/J mice treated with oligodendrocyte-reactive mAbs O1, O4, A2B5, and HNK-1 showed significantly enhanced CNS remyelination as compared with SJL/J mice treated with control IgM $\kappa$ or PBS (Table 2). Approximately $20-24 \%$ of the area of demyelination was remyelinated in mice treated with O1, O4, A2B5, and HNK-1. Remyelinated lesions in mice treated with O1, O4, A2B5, and HNK-1 were characterized by axons with thin myelin sheaths relative to axon diameter and a relative absence of inflammation (see Fig. 1). In contrast, mice treated with R24 did not show significantly enhanced remyelination (Table 2; Fig. 1). Of interest, these mAbs had no effect on the extent of demyelination and were not pathogenic (Table 2).

O1, O4, A2B5, HNK-1, and R24 did not react with any of the 


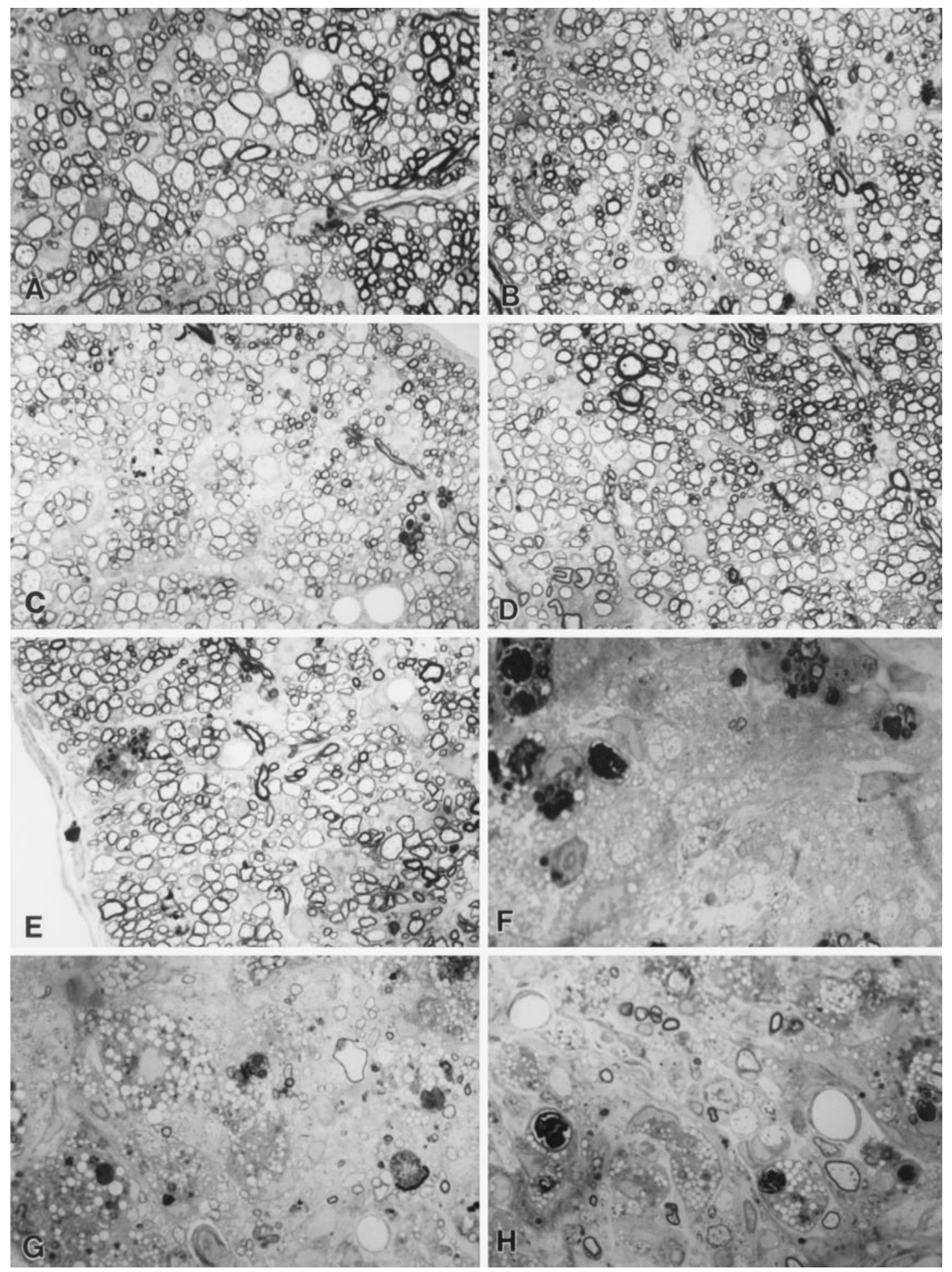

Figure 1. Light micrograph demonstrating extensive CNS remyelination after treatment with SCH79.08, O1, O4, A2B5, and HNK-1. Sections are from the spinal cords of SJL/J mice chronically infected with TMEV. Note the CNS remyelination, characterized by abnormally thin myelin sheath as compared with axon diameter, in demyelinated lesions of mice treated with SCH79.08 $(A), \mathrm{O} 1(B), \mathrm{O} 4(C), \mathrm{A} 2 \mathrm{~B} 5(D)$, and HNK-1 $(E)$. Note the demyelination without significant remyelination in mice treated with R24 $(F), \mathrm{CH} 12(G)$, and control IgM $\kappa$ ABPC22 $(H)$. Araldite-embedded sections were stained with $1 \%$ p-phenylenediamine (magnification, $875 \times$ ).

capsid proteins of TMEV, as confirmed by Western blotting (Fig. $4 A$ ). Immunocytochemical study showed that, although $\mathrm{R} 24$ reacted with the surface of O-2A progenitor cells, it did not stain intracellular structures of glial cells (see Fig. 2). By ELISA, R24 did not react with any of the protein antigens and chemical haptens that were examined (data not shown), indicating that R24 is not polyreactive in contrast to O1, O4, A2B5, and HNK-1 (Asakura et al., 1995). 

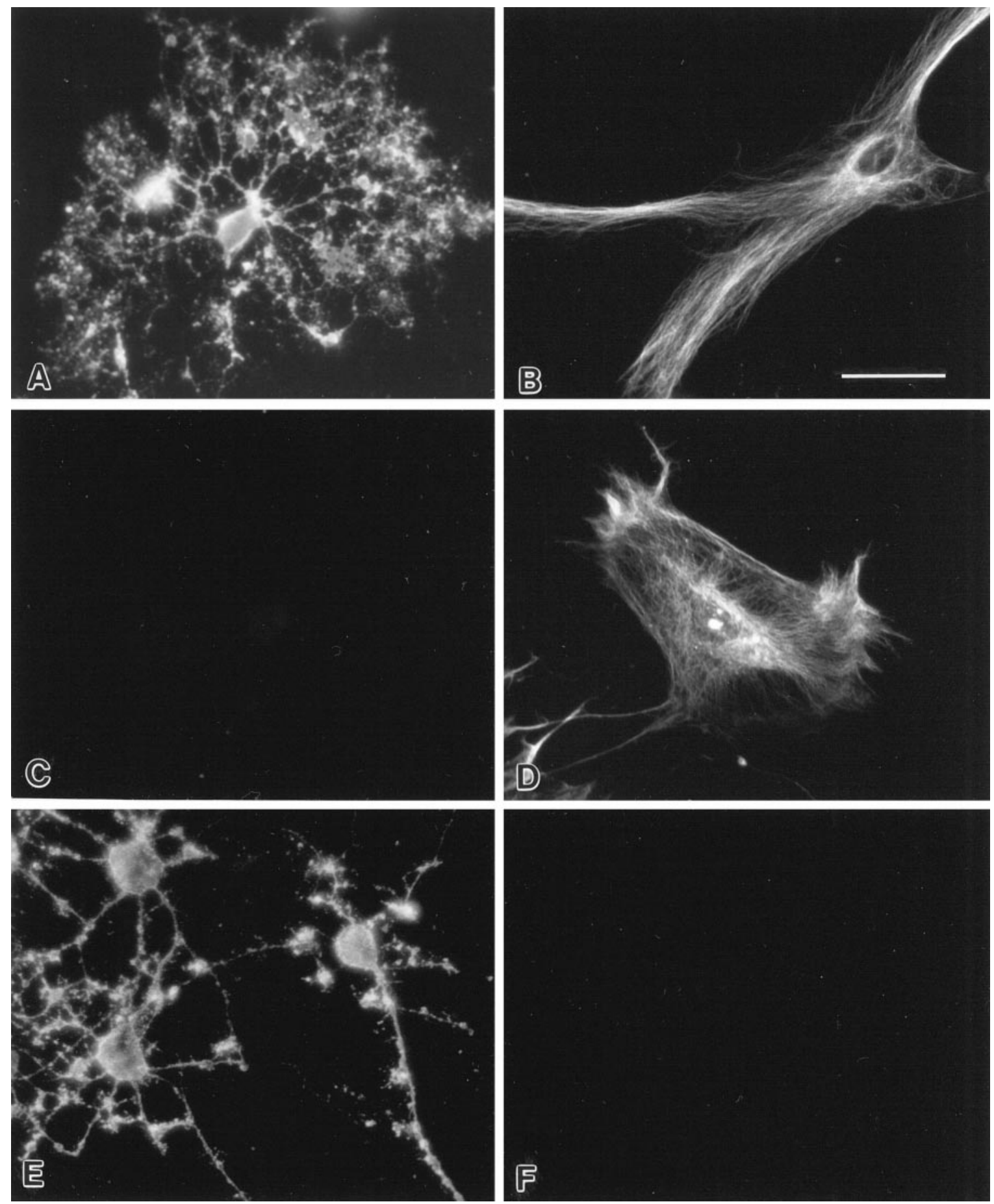

Figure 2. Indirect immunofluorescence of cultured glial cells. Note the live surface staining of oligodendrocytes with SCH94.03 $(A)$ and R24 $(E)$ but the absence of surface staining with SCH79.08 $(C)$. Also note intracellular staining of the cytoplasm of astrocytes with SCH94.03 $(B)$ and SCH79.08 $(D)$ but the absence of staining with R24 $(F)$. Scale bar, $20 \mu \mathrm{m}$. Oligodendrocytes and astrocytes were isolated from telencephalon of newborn Sprague Dawley rats.

\section{Complementarity-determining region 3 of $\mathrm{Ig}$ is crucial for enhanced remyelination}

Having established that a unique family of oligodendrocytereactive Abs, each with distinct surface antigen reactivities, would induce remyelination, it was critically important to determine whether the complementarity-determining regions (CDR), which form the Ab-binding site, are essential. Mouse B-cell lymphoma $\mathrm{CH} 12$ (CH12.Lx cell) secretes $\mathrm{IgM} \kappa \mathrm{mAb}$ under stimulation with lipopolysaccharide. This secreted $\operatorname{IgM} \kappa \mathrm{mAb} C H 12$ is encoded by exactly the same germline Ig genes as the genes of SCH94.03; the nucleotide and amino acid differences between SCH94.03 and CH12 exist only in the CDR3 of heavy and light chains (Miller and Rodriguez, 1995a). Despite this structural similarity, CH12 did not stain the surface of oligodendrocytes, did not stain intracellular structure of glial cells (data not shown), and was not polyreactive by ELISA (Miller and Rodriguez, 1995a). TMEV- 


\section{Protein antigens}

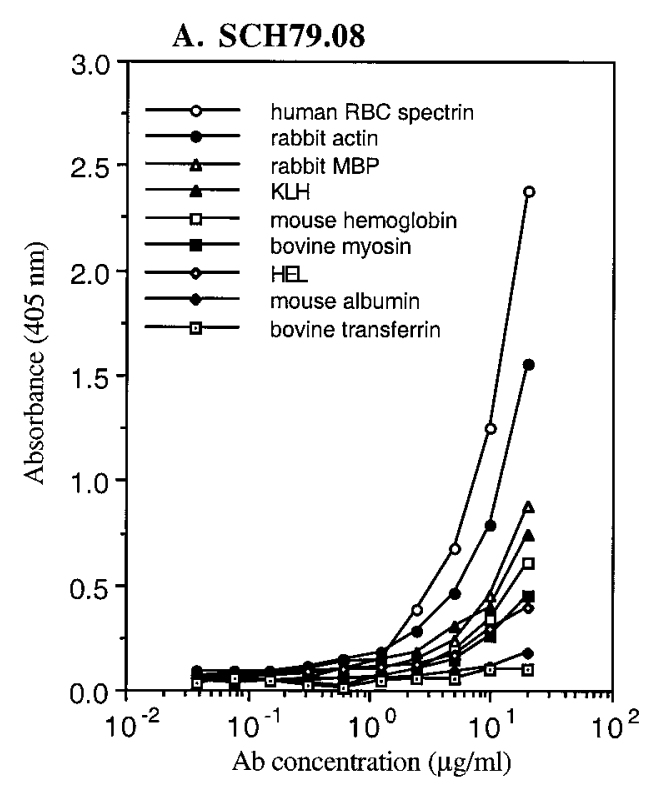

Chemical haptens

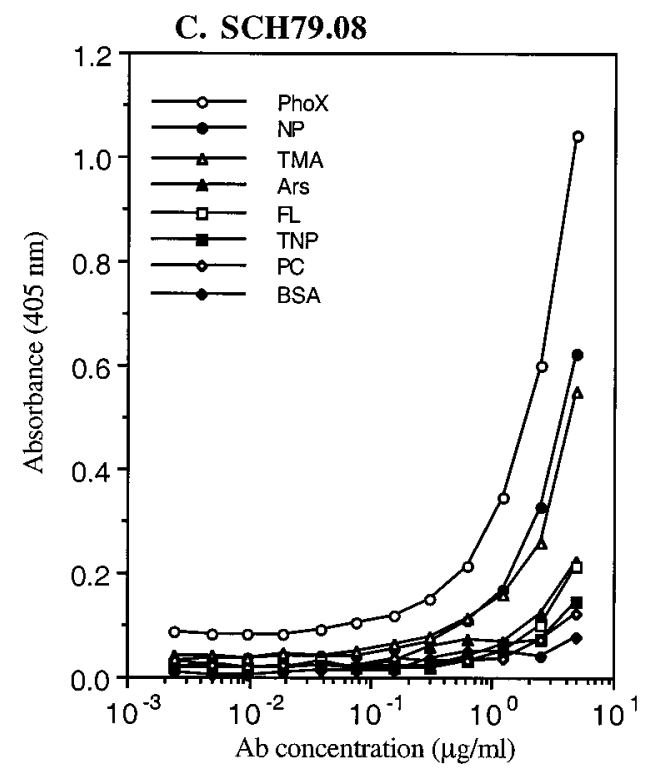

\section{B. control IgM (XMMEN-OE5)}

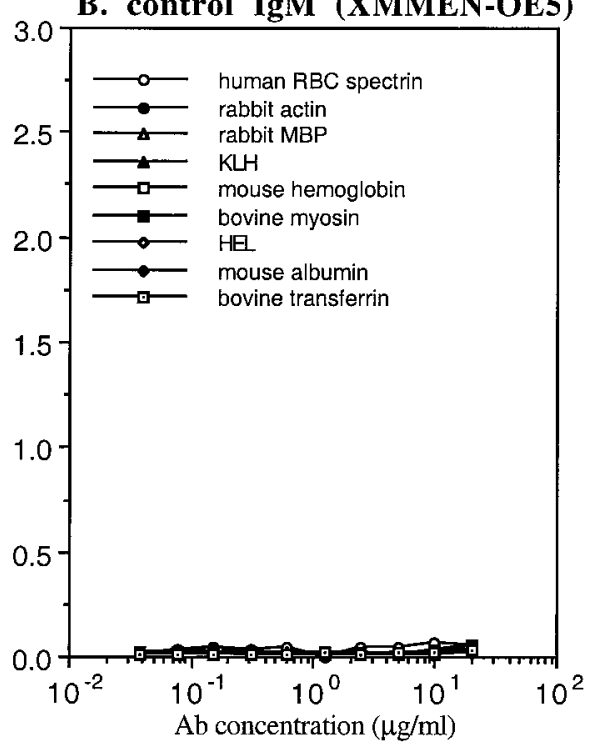

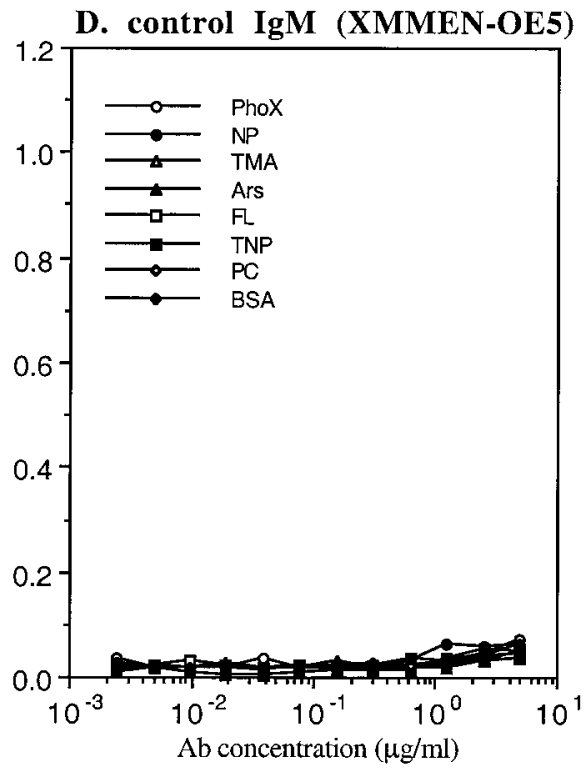

Figure 3. Protein antigen reactivity $(A)$ and chemical hapten reactivity $(C)$ of SCH79.08 are assessed by direct ELISA. Also shown are protein antigen reactivity $(B)$ and chemical hapten reactivity $(D)$ of control IgM (XMMEN-OE5). Abbreviations used in these panels: Ars, azophenylarsonate; $B S A$, bovine serum albumin; $F L$, fluorescein; $H E L$, hen egg lysozyme; $K L H$, keyhole limpet hemocyanin; $M B P$, myelin basic protein; $N P$, (4-hydroxy-3nitrophenyl)acetyl; PC, azophenylphosphoryl-choline; PhoX, phenyloxazolone; $R B C$, red blood cells; TMA, azophenyltrimethyl ammonium; TNP, trinytrophenyl acetyl. No reactivity to these protein antigens or chemical haptens was detected with another control IgM mAb (TEPC 183) (data not shown).

infected SJL/J mice treated with $\mathrm{CH} 12 \operatorname{IgM} \kappa \mathrm{mAb}$ did not show significant CNS remyelination when compared with control groups (Table 2; Fig. 1).

\section{DISCUSSION}

In this study we demonstrated that a panel of oligodendrocytereactive $\operatorname{IgM} \kappa \mathrm{mAbs}$ promotes remyelination in a virus model of MS. An $\operatorname{IgM} \kappa \mathrm{mAb} \mathrm{SCH} 79.08$, isolated from mice immunized with emulsions of homogenized normal mouse spinal cord, is polyreactive, binds to $\mathrm{MBP}$, and enhances myelin repair. In addition, other well recognized oligodendrocyte-reactive $\operatorname{IgM\kappa }$ mAbs (O1, O4, A2B5, and HNK-1) also promote CNS remyelination. The results support the hypothesis that the targeting of polyreactive Abs to oligodendrocytes has the potential to promote CNS remyelination.

Although cDNA sequence analysis revealed that variable regions of SCH79.08 are completely different from those of the prototypic remyelination-promoting $\mathrm{mAb} \mathrm{SCH} 94.03$ in both 


\section{A. Western blotting for TMEV}

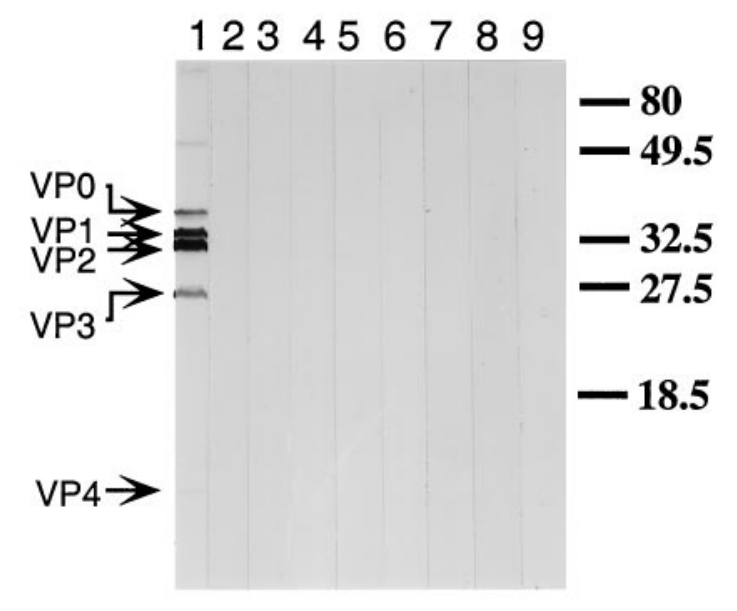

\section{B. Western blotting for MBP}

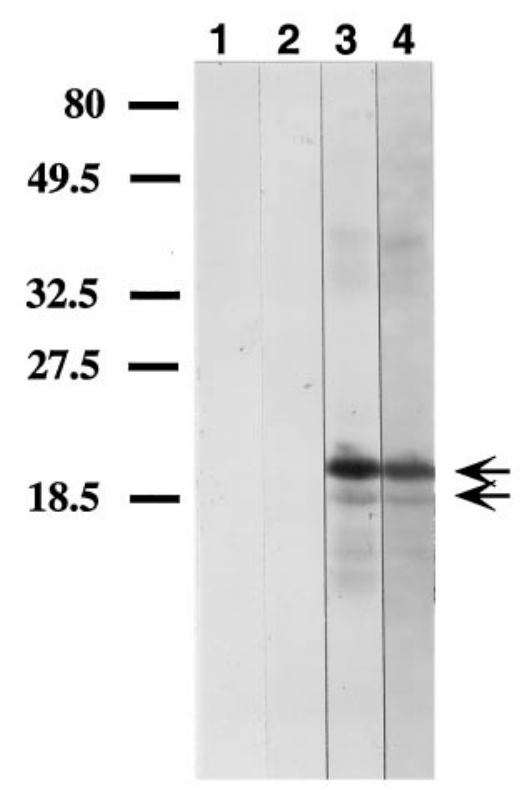

Figure 4. Western blotting of TMEV proteins $(A)$ and MBP $(B)$. Proteins from purified TMEV and rabbit MBP (obtained from Sigma) were separated on $15 \%$ SDS polyacrylamide gels. Bound Ig was detected with alkaline phosphatase-conjugated secondary antibodies by using BCIP/ NBT. Molecular weight markers are indicated in $\mathrm{kDa}$ at the right or left margin. A, Lane 1, Polyclonal rabbit anti-TMEV Ab; lane 2, SCH79.08; lane 3, O1; lane 4, O4; lane 5, A2B5; lane 6, HNK-1; lane 7, CH12; lane 8, R24; lane 9, control mouse IgM (MOPC 104E). Arrows indicate TMEV capsid proteins. B, Lane 1, Control mouse IgM (MOPC 104E); lane 2, $\mathrm{SCH} 94.03$; lane 3, polyclonal rabbit anti-MBP Ab (obtained from Dako); lane 4, SCH79.08. Arrows indicate two MBP isoforms (21.5 and $18.5 \mathrm{kDa})$ recognized by $\mathrm{SCH} 79.08$. heavy and light chains and that these mAbs were selected exclusively for their ability to promote remyelination and not on the basis of antigen specificity, this characterization showed that SCH79.08 has remarkable similarities with SCH94.03. Both mAbs (1) belong to the $\operatorname{IgM} \kappa$ subclass (Miller et al., 1994); (2) are multi-organ reactive (Miller et al., 1996) and polyreactive toward multiple protein antigens and chemical haptens by ELISA (Miller and Rodriguez, 1995a); (3) strongly stain cytoplasmic structures of most cells by immunofluorescence in a similar staining pattern of cytoskeletal proteins (Asakura et al., 1996b); and (4) recognize oligodendrocyte-specific autoantigens: SCH79.08 reacting with MBP and SCH94.03 binding to an uncharacterized surface antigen on oligodendrocytes (Asakura et al., 1996b). These results indicate that $\mathrm{SCH} 79.08$ has the characteristic features of a natural autoantibody, which is produced by autoreactive B-cells and is known to exist in healthy humans and rodents (Avrameas and Ternynck, 1993). The fact that both SCH79.08 and SCH94.03 bind to oligodendrocytes, but react to different antigens on these cells, supports the indirect hypothesis of Ab-mediated CNS remyelination.

Besides SCH79.08 and SCH94.03, other oligodendrocytereactive $\mathrm{IgM} \kappa \mathrm{mAbs} \mathrm{O} 1, \mathrm{O} 4, \mathrm{~A} 2 \mathrm{~B} 5$, and $\mathrm{HNK}-1$ also promote CNS remyelination in the TMEV model of MS. Despite the fact that all of the mAbs that promote CNS remyelination are $\operatorname{IgM} \kappa$, there is no obvious common pattern in germline Ig gene usage of these mAbs (Asakura et al., 1995). Although R24 binds to oligodendrocyte progenitors, it does not promote remyelination possibly because of its IgG isotype, lack of polyreactivity by immunocytochemistry and ELISA, or reactivity to oligodendrocytes of an earlier developmental stage. $\mathrm{CH} 12$, which has been classified as a natural autoantibody (Dighiero et al., 1987), was not widely polyreactive (Miller and Rodriguez, 1995a), did not bind to oligodendrocytes, and did not promote remyelination. Taken in concert, this indicates that only a unique population of polyreactive natural autoantibodies with oligodendrocyte reactivity, irrespective of antigen specificity, is effective for treatment. To this point only Igs of the $\mu$-isotypes have induced remyelination, suggesting the possibility that effector functions preferentially mediated by the $\mu$-heavy chain are central to this process. Additionally, the biological difference between SCH94.03 and $\mathrm{CH} 12$ establishes that the CDR3 of Ig is critical for the remyelinationpromoting activity for this group of Abs and supports the hypothesis that the ability of these Abs to bind within the demyelinated lesions is important to the induction of remyelination.

The mAbs that promote remyelination recognize different differentiation stages of oligodendrocytes from progenitor to mature, suggesting that remyelination-promoting activity may be independent of the developmental stage. The direct binding to surviving oligodendrocytes in the lesion could promote their dedifferentiation. Alternatively, the Abs could block the differentiation of oligodendrocytes to sustain their reactivity with growth factors. There is support from in vitro studies for these possibilities. MAb O4 was reported to stimulate differentiation of oligodendrocytes (Bansal et al., 1988). Abs to galactocerebroside cause transmembrane signaling in oligodendrocytes (Dyer and Benjamins, 1990). A mAb (R-mAb) that recognizes galactocerebroside, sulfatide, and a developmentally regulated unidentified antigen on oligodendrocytes reversibly blocks oligodendrocyte progenitor cell differentiation at the late progenitor stage (Bansal and Pfeiffer, 1989). In the presence of R-mAb, mature oligodendrocytes expressing terminally differentiated markers showed a retraction of processes, the formation of swollen cells, and a 
Table 2. In vivo effects of oligodendrocyte-reactive mAbs and CH12 IgMк mAb

\begin{tabular}{|c|c|c|c|c|c|}
\hline Treatment & $\begin{array}{l}\text { Number } \\
\text { of mice }\end{array}$ & $\begin{array}{l}\text { Area of white } \\
\text { matter }\left(\mathrm{mm}^{2}\right)\end{array}$ & $\begin{array}{l}\text { Area of demyelinated } \\
\text { lesion }\left(\mathrm{mm}^{2}\right)\end{array}$ & $\begin{array}{l}\text { Area of CNS-type } \\
\text { remyelination }\left(\mathrm{mm}^{2}\right)\end{array}$ & $\begin{array}{l}\text { Area of CNS-type } \\
\text { remyelination/area } \\
\text { of lesions }(\%)\end{array}$ \\
\hline $\mathrm{O} 1$ & 6 & $7.57 \pm 0.52$ & $0.53 \pm 0.10$ & $0.14 \pm 0.04$ & $24.8 \pm 6.2^{*}$ \\
\hline $\mathrm{O} 4$ & 7 & $8.01 \pm 0.16$ & $0.84 \pm 0.10$ & $0.17 \pm 0.05$ & $20.4 \pm 4.2^{*}$ \\
\hline A2B5 & 7 & $7.28 \pm 0.38$ & $0.70 \pm 0.18$ & $0.18 \pm 0.05$ & $24.6 \pm 4.6^{*}$ \\
\hline HNK-1 & 7 & $7.16 \pm 0.38$ & $0.78 \pm 0.10$ & $0.15 \pm 0.03$ & $20.6 \pm 2.8^{* *}$ \\
\hline CH12 & 7 & $7.43 \pm 0.28$ & $0.57 \pm 0.11$ & $0.05 \pm 0.02$ & $8.5 \pm 4.6$ \\
\hline $\mathrm{R} 24$ & 7 & $7.52 \pm 0.34$ & $0.65 \pm 0.13$ & $0.04 \pm 0.01$ & $6.7 \pm 2.5$ \\
\hline XMMEN-OE5 & 6 & $9.32 \pm 0.42$ & $1.06 \pm 0.27$ & $0.03 \pm 0.01$ & $3.4 \pm 1.0$ \\
\hline PBS & 6 & $7.46 \pm 0.76$ & $0.51 \pm 0.15$ & $0.05 \pm 0.02$ & $8.0 \pm 2.2$ \\
\hline
\end{tabular}

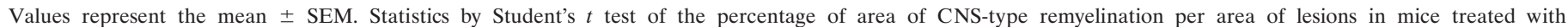

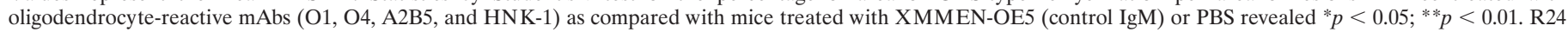

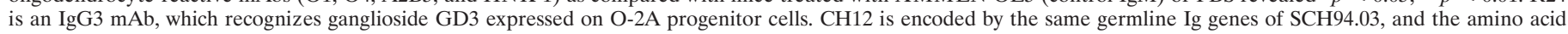
differences between them exist only in the CDR3.

reduction of the levels of terminally differentiated markers (Bansal and Pfeiffer, 1994). MAG, which is recognized by HNK-1, has been shown to be a major inhibitor of axonal regeneration in vitro (McKerracher et al., 1994; Mukhopadhyay et al., 1994), although its inhibitory activity of axonal regeneration in vivo remains inconclusive (Bartsch et al., 1995; Schäfer et al., 1996). Possibly, HNK-1 may promote CNS remyelination by interfering with MAG expression.

The observation that multiple oligodendrocyte-reactive Abs, each with distinct antigen specificities, promote remyelination is most consistent with the hypothesis that direct binding of the mAbs to injured oligodendrocytes in the lesion induces myelin repair via an immune effector mechanism initiated by the $\mu$-heavy chain. Consistent with this hypothesis, we previously reported that affinity-purified polyclonal anti-MBP Abs promote CNS remyelination; therefore, reactivity to an intracellular marker of mature oligodendrocytes is also effective for myelin repair (Rodriguez et al., 1996). In further support of the hypothesis, all mAbs that promoted remyelination not only bound to oligodendrocytes but also reacted with intracellular antigens. One possibility is that IgM binding to damaged cells enhances their removal by scavenger macrophages and microglia so that healthy oligodendrocytes or O-2A progenitor cells can initiate their myelination program.

\section{REFERENCES}

Abo T, Balch CM (1981) A differentiation antigen of human NK and K cells identified by a monoclonal antibody (HNK-1). J Immunol 127:1024-1029.

Armstrong RC, Dorn HH, Kufta CV, Friedman E, Dubois-Dalcq ME (1992) Pre-oligodendrocyte from adult human CNS. J Neurosci 12:1538-1547.

Asakura K, Miller DJ, Pogulis RJ, Pease LR, Rodriguez M (1995) Oligodendrocyte-reactive O1, O4, and HNK-1 monoclonal antibodies are encoded by germline immunoglobulin genes. Mol Brain Res 34:283-293.

Asakura K, Pogulis RJ, Pease LR, Rodriguez M (1996a) A monoclonal autoantibody which promotes central nervous system remyelination is highly polyreactive to multiple known and novel antigens. J Neuroimmunol 65:11-19.

Asakura K, Miller DJ, Murray K, Bansal R, Pfeiffer SE, Rodriguez M (1996b) Monoclonal autoantibody SCH94.03, which promotes CNS remyelination, recognizes an antigen on the surface of oligodendrocytes. J Neurosci Res 43:273-281.

Avrameas S, Ternynck T (1993) The natural autoantibodies system: between hypotheses and facts. Mol Immunol 30:1133-1142.
Bansal R, Pfeiffer SE (1989) Reversible inhibition of oligodendrocyte progenitor differentiation by a monoclonal antibody against surface galactolipids. Proc Natl Acad Sci USA 86:6181-6185.

Bansal R, Pfeiffer SE (1994) Regulation of gene expression in mature oligodendrocytes by the specialized myelin-like membrane environment: antibody perturbation in culture with the monoclonal antibody R-mAb. Glia 12:173-179.

Bansal R, Pfeiffer SE (1997) FGF-2 converts mature oligodendrocytes to a novel phenotype. J Neurosci Res 50:215-228.

Bansal R, Gard AL, Pfeiffer SE (1988) Stimulation of oligodendrocyte differentiation in culture by growth in the presence of a monoclonal antibody to sulfated glycolipid. J Neurosci Res 21:260-267.

Bansal R, Warrington AE, Gard AL, Ranscht B, Pfeiffer SE (1989) Multiple and novel specificities of monoclonal antibodies O1, O4, and $\mathrm{R}-\mathrm{mAb}$ used in the analysis of oligodendrocyte development. J Neurosci Res 24:548-557.

Bansal R, Stefansson K, Pfeiffer SE (1992) Proligodendroblast antigen (POA), a developmental antigen expressed by A007/O4-positive oligodendrocyte progenitors prior to the appearance of the sulfatide and galactocerebroside. J Neurochem 58:2221-2229.

Bartsch U, Bandtlow CE, Schnell L, Bartsch S, Spillmann AA, Rubin BP, Hillenbrand R, Montag D, Schwab ME, Schachner M (1995) Lack of evidence that myelin-associated glycoprotein is a major inhibitor of axonal regeneration in the CNS. Neuron 15:1375-1381.

Dighiero G, Poncet P, Matthes T, Kaushik A (1987) Is autoantibody production related to particular B-cell subsets and variable region genes? Pathol Immunopathol Res 6:371-389.

Dyer CA, Benjamins JA (1990) Glycolipids and transmembrane signaling: antibodies to galactocerebroside cause an influx of calcium in oligodendrocytes. J Cell Biol 111:625-633.

Eisenbarth GS, Walsh FS, Nirenberg M (1979) Monoclonal antibody to a plasma membrane antigen of neurons. Proc Natl Acad Sci USA 76:4913-4917.

Fazekas F, Deisenhammer F, Strasser-Fuchs S, Nahler G, Mamoli B (1997) Randomized placebo-controlled trial of monthly intravenous immunoglobulin therapy in relapsing-remitting multiple sclerosis. Lancet 349:589-593.

Fredman P, Magnani JL, Nirenberg M, Ginsburg V (1984) Monoclonal antibody A2B5 reacts with many gangliosides in neuronal tissue. Arch Biochem Biophys 233:661-666.

Fressinaud C, Laeng P, Labourdette G, Durand J, Vallat J-M (1993) The proliferation of mature oligodendrocytes in vitro is stimulated by basic fibroblast growth factor and inhibited by oligodendrocyte-type 2 astrocyte precursors. Dev Biol 158:317-329.

Grinspan JB, Stern JL, Franceschini B, Pleasure D (1993) Trophic effects of basic fibroblast growth factor (bFGF) on differentiated oligodendroglia: a mechanism for regeneration of the oligodendroglial lineage. J Neurosci Res 36:672-680.

Kasai N, Yu RK (1983) The monoclonal antibody A2B5 is specific to ganglioside GQ1c. Brain Res 277:155-158.

McGarry RC, Helfand SL, Quarles RH, Roder JC (1983) Recognition of 
myelin-associated glycoprotein by the monoclonal antibody HNK-1. Nature 306:376-378.

McKerracher L, David S, Jackson DL, Kottis V, Dunn RJ, Braun PE (1994) Identification of myelin-associated glycoprotein as a major myelin-derived inhibitor of neurite growth. Neuron 13:805-811.

Miller DJ, Rodriguez M (1995a) A monoclonal autoantibody that promotes central nervous system remyelination in a model of multiple sclerosis is a natural autoantibody encoded by germline immunoglobulin genes. J Immunol 154:2460-2469.

Miller DJ, Rodriguez M (1995b) Spontaneous and induced remyelination in multiple sclerosis and the Theiler's virus model of central nervous system demyelination. Microsc Res Tech 32:230-245.

Miller DJ, Sanborn KS, Katzmann JA, Rodriguez M (1994) Monoclonal antibodies promote central nervous system repair in an animal model of multiple sclerosis. J Neurosci 14:6230-6238.

Miller DJ, Njenga MK, Parisi JE, Rodriguez M (1996) Multi-organ reactivity of a monoclonal natural autoantibody that promotes remyelination in a mouse model of multiple sclerosis. J Histochem Cytochem 44:1005-1011.

Mukhopadhyay G, Doherty P, Walsh FS, Crocker PR, Filbin MT (1994) A novel role for myelin-associated glycoprotein as an inhibitor of axonal regeneration. Neuron 13:757-767.

Njenga MK, Pavelko KD, Baisch J, Lin X, David C, Leibowitz J, Rodriguez M (1996) Theiler's virus persistence and demyelination in major histocompatibility complex class II-deficient mice. J Virol 70:1729-1737.

Noseworthy JH, O’Brien PC, van Engelen BGM, Rodriguez M (1994) Intravenous immunoglobulin therapy in multiple sclerosis: progress from remyelination in the Theiler's virus model to a randomised, double-blind, placebo-controlled clinical trial. J Neurol Neurosurg Psychiatry 57[Suppl]:11-14.

Prineas JW, Connell F (1979) Remyelination in multiple sclerosis. Ann Neurol 5:22-31.

Rodriguez M, Miller DJ, Lennon VA (1996) Immunoglobulins reactive with myelin basic protein promote CNS remyelination. Neurology 46:538-545.

Schäfer M, Fruttiger M, Montag D, Schachner M, Martini R (1996) Disruption of the gene for the myelin-associated glycoprotein improves axonal regrowth along myelin in C57BL/Wlds mice. Neuron 16:1107-1113.

Sommer I, Schachner M (1981) Monoclonal antibodies (O1 to O4) to oligodendrocyte cell surfaces: an immunocytological study in the central nervous system. Dev Biol 83:311-327.

Wolswijk G, Noble M (1989) Identification of an adult-specific glial progenitor cell. Development 105:387-400.

Wood PM, Bunge RP (1991) The origin of remyelinating cells in adult central nervous system: the role of the mature oligodendrocyte. Glia 4:225-232. 